\title{
PURIFICAÇÃO DE GLICERINA UTILIZANDO MEMBRANAS CERÂMICAS
}

\author{
J. F. MEDEIROS ${ }^{1 *}$, V. Y. KAJIHARA ${ }^{1}$, J. V. BERNI ${ }^{1}$, F. N. de ALMEIDA ${ }^{1}$, M. C. S. \\ GOMES $^{2}$, N. C. PEREIRA ${ }^{1}$ \\ ${ }^{1}$ Universidade Estadual de Maringá, Departamento de Engenharia Química \\ ${ }^{2}$ Universidade Tecnológica Federal do Paraná, Departamento de Engenharia Química \\ e-mail: janainafmedeiros1@ hotmail.com
}

\begin{abstract}
RESUMO
A intensa busca por fontes de energias renováveis e processos sustentáveis visando à redução da poluição ambiental e do aquecimento do planeta, tem estimulado o mercado mundial de biocombustíveis. O biodiesel possui destaque na matriz energética nacional e sua produção em larga escala gera grandes quantidades de glicerina com altos teores de impureza. $\mathrm{O}$ aumento da oferta de glicerina impura no mercado leva à necessidade de novas metodologias para sua purificação levando à viabilização do custo final do biodiesel. Este trabalho tem como objetivo o estudo da purificação de glicerina industrial, utilizando processos com membranas de ultrafiltração com diâmetro de poros de $5 \mathrm{kDa}, 20 \mathrm{kDa}$ e $0,05 \mu \mathrm{m}$, em pressões de 1,2 e 3 bar a uma temperatura de $60^{\circ} \mathrm{C}$. Os resultados demonstraram que o maior teor de glicerol no permeado $(89,6 \%)$ foi obtido utilizando a membrana de $5 \mathrm{kDa}$ na pressão de 3 bar.
\end{abstract}

\section{INTRODUÇÃO}

As mudanças climáticas, induzidas em grande parte pelo uso de combustíveis fósseis, associadas à preocupação com o desenvolvimento sustentável, tornaram extremamente necessárias as fontes renováveis de energia. No Brasil e no mundo, a demanda por sistemas energéticos que se enquadrem nas metas estabelecidas pelo Protocolo de Kyoto tem gerado a necessidade de substituir os combustíveis fósseis, maiores geradores de gases de efeito estufa, por fontes derivadas da energia da biomassa (VISENTAINER, 2013).

A utilização de biodiesel, em substituição aos combustíveis fósseis é uma alternativa que promove benefícios ambientais, uma vez que, por não conter enxofre e compostos aromáticos, proporciona uma queima mais limpa, sem a formação de dióxido de enxofre e compostos cancerígenos. Além disso, o biodiesel apresenta uma composição química homogênea com a presença de oxigênio, o que contribui para uma combustão mais eficiente (KNOTHE et al., 2006).

Segundo Visentainer (2013), o biodiesel representa uma alternativa energética que traz uma série de vantagens: equilibra o balanço negativo gerado pela emissão de $\mathrm{CO}_{2}$ na atmosfera, reduzindo desta forma o aquecimento global; permite a valorização de subprodutos de atividades agroindustriais; aumenta a fixação do homem no campo; reduz a emissão de compostos sulfurados 
(SOx) e materiais particulados, quando comparado ao diesel.

Essas vantagens podem explicar o crescimento da produção de biodiesel no Brasil. Desde o dia $1^{\circ}$ de novembro de 2014, o óleo diesel comercializado no país contém $7 \%$ de biodiesel, isto é, um aumento de $2 \%$ em comparação com a legislação anterior, estabelecido pelo Conselho Nacional de Política Energética (CNPE).

Essa crescente demanda de biodiesel tem ocasionado uma elevação na produção dos coprodutos, sendo a glicerina um dos principais (MOTA et al., 2009). Segundo Tan et al. (2013) para a produção de $10 \mathrm{~kg}$ de biodiesel por meio do processo de transesterificação produz-se aproximadamente $1 \mathrm{~kg}$ de glicerol bruto.

Segundo Santos (2009) o Brasil ao produzir biodiesel para adição de 5\% no óleo diesel (aproximadamente 2,6 milhões de toneladas do biocombustível por ano) gerava em média quase $300 \mathrm{mil}$ toneladas de glicerina por ano. No entanto, esta quantidade não é totalmente absorvida e o excedente de glicerina vem saturando o mercado, sendo sua oferta maior que a procura, levando assim a queda do preço desse subproduto e a estocada do mesmo sem um destino certo.

Embora o glicerol possa ser produzido por meio de várias vias (saponificação, hidrólise, esterificação e transesterificação), sua produção em massa é alcançada atualmente por meio do processo de transesterificação (TAN et al., 2013). Sendo assim a maior parte do glicerol disponível é um subproduto da produção de biodiesel, que está contido na fase de glicerol em conjunto com muitos outros materiais, tais como sabões, catalisador restante, água e ésteres formados durante o processo (HÁJEK et al., 2010).

O glicerol (1,2,3 propanotriol) foi descoberto por Scheele em 1779 durante o processo de saponificação de azeite de oliva. É um poliálcool de fórmula estrutural apresentada na Figura 1 o qual está presente em diferentes espécies (ARRUDA et al., 2007).

Figura 1 - Fórmula estrutural do glicerol.

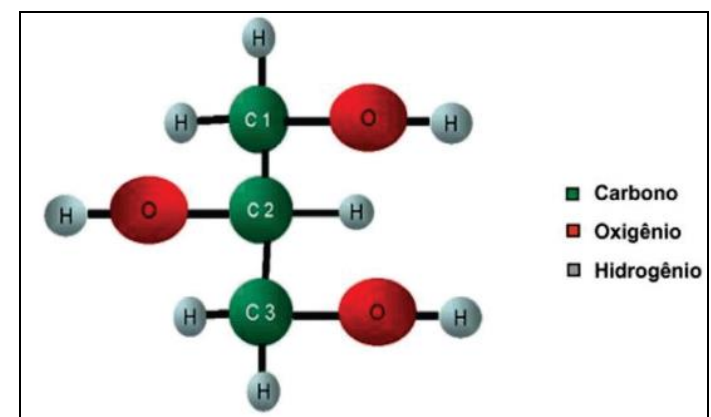

Fonte: (ARRUDA et al., 2007)

Glicerol ou propano-1,2,3-triol (IUPAC) é um composto orgânico pertencente à função álcool sendo à temperatura ambiente $\left(25^{\circ} \mathrm{C}\right)$, líquido, higroscópico, inodoro, viscoso e de sabor adocicado. O termo glicerina refere-se à solução de glicerol. (KNOTHE, 2005).

Segundo Ayoub et al. (2012) o glicerol é um substrato não tóxico, biodegradável e versátil, que pode ser convertido em vários produtos químicos ou biológicos.

Atualmente, a glicerina é empregada em vários ramos industriais. Sua maior aplicação é em indústrias de medicamentos, produtos de higiene bucal e indústria de cosméticos (MOTA et al. 2009).

Segundo OOI et al. (2004) o valor do glicerol bruto obtido da produção de biodiesel encontra-se entre 0,2 a $0,4 \mathrm{R} \$ / \mathrm{kg}$. Este baixo valor é atribuído ao conteúdo de aproximadamente $30 \%(\mathrm{p} / \mathrm{p})$ de impurezas e ao grande volume deste co-produto gerado pelas indústrias.

Por este motivo, de acordo com Tan et al. (2013), várias técnicas de purificação de glicerol estão sendo desenvolvidas, como por exemplo, a utilização de carvão ativado, de resinas de permuta iônica visando obter um glicerol com alto grau de pureza que ajudará a elevar os padrões de atividades do biodiesel. 
Gomes et al. (2013) estudou o uso de adição de água acidificada na purificação de biodiesel por microfiltração e seus resultados mostraram um grande efeito na remoção de glicerol da fase de biodiesel. Esta remoção ocorre porque a adição de água promove a formação de uma fase aquosa, incluindo glicerina, sabão e etanol, formando aglomerados com tamanho médio maior do que o diâmetro dos poros da membrana.

Amin et al. (2010) estudou o efeito dos ácidos graxos na incrustação de membranas de ultrafiltração e constatou que o ácido oleico com a cadeia de carbono mais longa contribuiu para um declínio de fluxo grave com a membrana em estudo. $\mathrm{O} \mathrm{pH}$ da mistura também desempenhou um papel importante na adsorção de ácidos graxos sobre a superfície da membrana, mostrando que em meios ácidos ocorreu uma grave redução do fluxo. Com base nestes resultados, é importante considerar a presença de ácidos graxos, pois podem causar uma diminuição considerável do fluxo no processo de ultrafiltração.

A utilização de processos com membranas é um método promissor para a purificação do glicerol, porem ainda é pouco significativo na área de biocombustíveis, e a escassez de trabalhos indica a necessidade do desenvolvimento de rotas de purificação mais eficientes.

Deste modo, o objetivo deste trabalho é o estudo da purificação da glicerina obtida como coproduto do processo de produção de biodiesel por meio de ultrafiltração com membranas cerâmicas.

\section{MATERIAIS E MÉTODOS}

A glicerina industrial foi doada pela indústria produtora de biodiesel BSBIOS localizada em Marialva - Paraná, sendo proveniente da produção de biodiesel com óleo de soja (70\%) e gordura animal (30\%).
A caracterização da glicerina bruta industrial e da glicerina PA (para posterior comparação de resultados) consistiu nas análises de massa específica, viscosidade cinemática, teor de umidade, ph, índice de acidez, remoção de cor e teor de glicerol, descritas a seguir.

\subsection{Massa específica}

Para a determinação da massa específica a $25^{\circ} \mathrm{C}$, foi utilizado um densímetro digital da marca Anton Paar modelo DMA 5000 do Departamento de Física da Universidade Estadual de Maringá.

\subsection{Viscosidade cinemática}

A viscosidade a $25^{\circ} \mathrm{C}$ foi determinada utilizando-se um reômetro digital da marca Brookfield modelo DV-III com spindle SC427 e velocidades rotacionais entre 10 e $220 \mathrm{rpm}$.

\subsection{Teor de umidade}

O teor de umidade foi determinado por meio do equipamento Karl Fischer da marca Analyser, modelo Umidade Controle KF1000 .

\subsection{Potencial Hidrogeniônico (pH)}

A medição do $\mathrm{pH}$ foi feita com auxílio de um pHmetro devidamente calibrado, e utilizou-se alíquotas de aproximadamente $30 \mathrm{~mL}$ das amostras para a determinação.

\section{5. Índice de acidez}

Para análise do índice de acidez (I.A) foi seguida a metodologia recomendada pela AOCS (Ca 5-40) com pequenas adaptações, utilizada por Viesser (2010). O método consiste em pesar 0,5 gramas da amostra de glicerina em um erlenmeyer de $125 \mathrm{ml}$ e adicionar $25 \mathrm{ml}$ de água e agitar até completa diluição. Logo após adicionar $1 \mathrm{ml}$ de fenolftaleína $1 \%$ e titular com solução de hidróxido de sódio $0,01 \mathrm{M}$ até o surgimento da coloração rósea permanente. Concomitante às 


\section{ENEMP \\ CONGRESSO BRASILEIRO \\ DE SISTEMAS PARTICULADOS \\ São Carlos - SP}

análises foi procedido um teste em branco. Para a determinação do índice de acidez foi utilizada a Equação 1:

Índice de acidez $(I A)=\frac{\left(V-V_{b}\right) \cdot M \cdot 40}{W}$

\subsection{Remoção de cor}

Foi construída uma curva de calibração variando-se a concentração de glicerina bruta de 0 a $100 \%$, dentro da solução de glicerina PA. Logo após foi realizado a varredura (190 a $1100 \mathrm{~nm})$ dessas amostras em espectrofotômetro da marca Thermo Scientific, modelo Genesys 10 UV Scanning. Por meio dessas varreduras foi definido 0 comprimento de onda a ser utilizado para a leitura das amostras.

\subsection{Teor de Glicerol}

Ferreira (2009), Rocha (2009) e Lopes (2014) escolheram o método do periodato para a determinação do teor de glicerol. Neste método o glicerol na presença de periodato é oxidado a ácido fórmico segundo a reação química apresentada a seguir:

$\mathrm{C}_{\mathrm{a}} \mathrm{H}_{\mathrm{a}} \mathrm{O}_{\mathrm{a}}+2 \mathrm{IO}^{-} \leftrightarrow 2 \mathrm{OO}_{\mathrm{a}}^{-}+2 \mathrm{CH}_{2} \mathrm{O}+\mathrm{HCOOH}+\mathrm{H}_{2} \mathrm{O}$

O ácido fórmico formado, que é equivalente ao glicerol existente na amostra, pode ser determinado por titulação ácidobase, utilizando um indicador e/ou medidor de pH para detectar o ponto final da titulação (NAVIGLIO et al., 2007).

O método consiste na diluição da amostra previamente pesada, aproximadamente $0,1 \mathrm{~g}$, em $5 \mathrm{~mL}$ de água destilada, adição de 2 a 3 gotas de azul de bromotimol, acidificação com ácido sulfúrico (H2SO4) $0,1 \mathrm{~mol} / \mathrm{L}$, até $\mathrm{pH} 4$, neutralização com hidróxido de sódio $(\mathrm{NaOH}) \quad 0,05 \mathrm{~mol} / \mathrm{L}$ até o aparecimento da coloração azul e adição de $10 \mathrm{~mL}$ de periodato de sódio $60 \mathrm{~g} / \mathrm{L}$. A amostra deverá ser mantida no escuro por 30 minutos em seguida adicionados $1 \mathrm{~mL}$ de etilenoglicol $99,5 \%$ e a mesma deverá ser mantida no escuro por mais 20 minutos. Logo após, a amostra será diluída a um volume de $30 \mathrm{~mL}$ com água destilada e a titulação realizada com hidróxido de sódio $0,125 \mathrm{~mol} / \mathrm{L}$, sob agitação magnética, com auxílio de um pHmetro para determinação do pH final de titulação, que deve ser de 6,5 para o branco e 8,1 para a amostra (FERREIRA, 2009). O percentual de glicerol será determinado pela Equação 2:

Glicerol $\left(\% \frac{m}{m}\right)=\frac{M M_{\text {gliceroI } N . f .} \cdot\left(V_{1}-V_{2}\right)}{m} \times 100$

\subsection{Experimentos de ultrafiltração}

Nos experimentos de ultrafiltração, foram utilizadas 3 membranas cerâmicas de $\alpha-\mathrm{Al}_{2} \mathrm{O}_{3} / \mathrm{TiO}_{2}$ (Shumacher GmbH-Ti 01070) adquiridas da NETZSCH. Duas das membranas são do tipo tubular monocanal, com comprimento de $250 \mathrm{~mm}$, diâmetro de 7 $\mathrm{mm}$ e área de filtração de $0,005 \mathrm{~m}^{2}$, com diâmetro médio de poros de $20 \mathrm{kDa}$ e $0,05 \mu \mathrm{m}$, como mostra a Figura 2(a). A outra membrana é multicanal com área de filtração de $0,011 \mathrm{~m}^{2}$ e com diâmetro médio de poros de $5 \mathrm{kDa}$, como mostra a Figura 2(b).

Figura 2 - Membranas cerâmicas tubulares de $20 \mathrm{kDa}$ e $0,05 \mu \mathrm{m}$ (a) e membrana cerâmica multicanal de $5 \mathrm{kDa}(\mathrm{b})$.

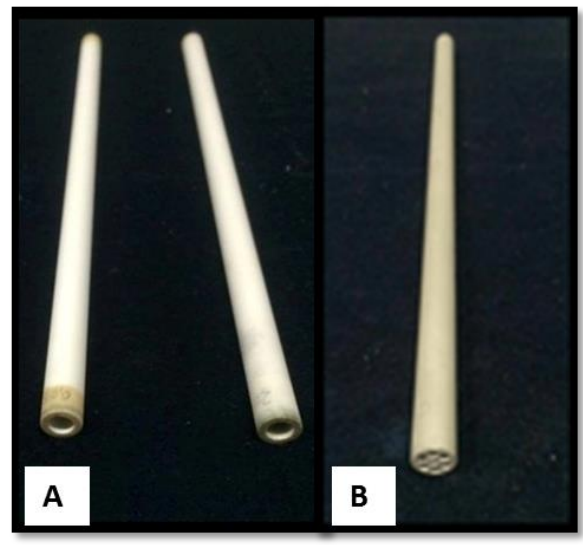


O equipamento experimental é uma unidade piloto de micro e ultrafiltração com filtração tangencial, da marca UF NETZSCH modelo 027.06-1C1/07-0005/AI, instalada no Laboratório de Processos de Separação II DEQ/UEM, como mostra a Figura 4.

Figura 3 - Unidade piloto de micro e ultrafiltração (vista frontal).



Para a purificação das amostras, em cada experimento, 3 a 4 litros da glicerina foram aquecidos até $60^{\circ} \mathrm{C}$ no tanque de alimentação e então bombeada para a tubulação. A pressão foi ajustada por meio de uma válvula manual e a temperatura controlada por um banho termostático. O permeado foi coletado e o concentrado totalmente recirculado para o tanque de alimentação. O tempo de duração de cada experimento foi de 90 minutos após o inicio da coleta de permeado.

Após cada processamento, a unidade experimental foi imediatamente limpa com recirculações de água e água com detergente a fim de evitar futuras contaminações. A limpeza das membranas foi realizada em banho ultrassônico com água, solução aquecida de $\mathrm{NaOH}(2 \%)$ e solução aquecida de ácido cítrico (2\%). Foi avaliada a permeabilidade hidráulica da membrana para que um parâmetro de limpeza pudesse ser estabelecido e após cada ciclo de regeneração foi medido o fluxo com água deionizada, garantindo a reprodutibilidade dos experimentos.
O fluxo permeado foi obtido por meio da determinação da massa de permeado coletada em função do tempo, medida em uma balança semi-analítica (BG 4000Gehaka), e calculado de acordo com a Equação 3.

$J_{\text {perm }}=\frac{m_{p}}{A . t}$

Após as filtrações as amostras de permeado e concentrado foram caracterizadas por meio da análise de teor de glicerol, descrita anteriormente.

\section{RESULTADOS}

\subsection{Caracterização das glicerinas}

A caracterização consistiu em analisar os parâmetros físico-químicos das glicerinas PA e bruta industrial, antes de qualquer purificação. Na Tabela 1 estão apresentados os resultados da caracterização.

Tabela 1 - Caracterização inicial da glicerina PA e bruta industrial.

\begin{tabular}{lcc}
\multicolumn{1}{c}{$\begin{array}{c}\text { Análises } \\
\text { experimentais }\end{array}$} & PA & $\begin{array}{c}\text { Bruta } \\
\text { industrial }\end{array}$ \\
\hline Acidez $(\mathrm{mgKOH} / \mathrm{g})$ & $0,26 \pm 0,13$ & $3,09 \pm 0,27$ \\
Umidade $(\%)$ & $1,32 \pm 0,01$ & $9,96 \pm 0,05$ \\
$\mathrm{pH}$ & $3,98 \pm 0,03$ & $6,37 \pm 0,11$ \\
$\begin{array}{l}\text { Massa específica } \\
\left(\mathrm{g} / \mathrm{cm}^{3}\right)\end{array}$ & $1,26 \pm 0,00$ & $1,27 \pm 0,00$ \\
$\begin{array}{l}\text { Teor de glicerol } \\
(\%)\end{array}$ & $98,0 \pm 5,0$ & $78,9 \pm 0,6$ \\
$\begin{array}{l}\text { Viscosidade } \\
\text { cinemática }\left(\mathrm{mm}^{2} / \mathrm{s}\right)\end{array}$ & 548,62 & 216,34 \\
\hline
\end{tabular}

Conforme expresso na Tabela 1, a glicerina industrial apresentou uma acidez e um $\mathrm{pH}$ maior que a glicerina PA indicando a presença de ácidos graxos não reagidos durante a produção de biodiesel. A umidade também está com uma porcentagem maior para a glicerina industrial, devido ao prétratamento realizado pela empresa, que 
consiste em acidificação, neutralização e secagem (possivelmente, não eficiente) da glicerina. Esta umidade elevada provoca uma diminuição da viscosidade da glicerina industrial, que está abaixo do esperado. A massa específica da glicerina industrial já se encontra com o mesmo valor da PA, não sendo necessário um tratamento para adequála. O teor de glicerol é o fator principal desta purificação sendo necessário alcançar no mínimo 95\% de glicerol.

Após a caracterização da glicerina, foram realizadas as purificações com membranas cerâmicas. Nas Figuras 5, 6 e 7, estão os fluxos de permeado (obtidos por meio da Equação 3), em função do tempo de ultrafiltração.

Figura 4-Gráfico de fluxo de permeado versus tempo, na pressão de 1 bar.

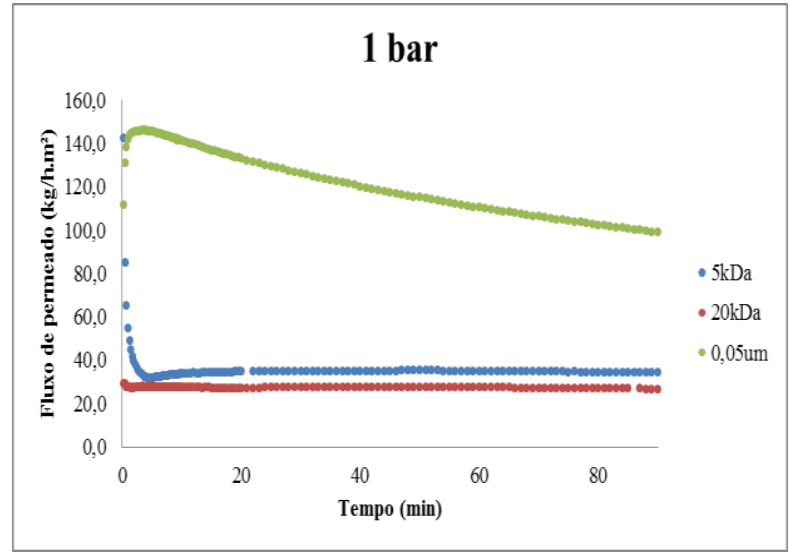

$\mathrm{Na}$ Figura 5 pode-se perceber que a membrana de $20 \mathrm{kDa}$ atingiu um equilíbrio rapidamente, pois o menor diâmetro de poros, provoca um menor entupimento e com isso um menor fouling, tornando o fluxo permeado estabilizado desde o início.

A membrana de $5 \mathrm{kDa}$ é multicanal, o diâmetro do canal é menor, não permitindo a comparação com as de monocanal. Apesar de um fluxo inicial elevado, pela maior área superficial, o fouling foi muito alto, de modo que o fluxo permeado estabilizado ficou próximo do obtido com a membrana de $5 \mathrm{kDa}$.
Figura 5 - Gráfico de fluxo de permeado versus tempo, na pressão de 2 bar.



Figura 6 - Gráfico de fluxo de permeado versus tempo, na pressão de 3 bar.

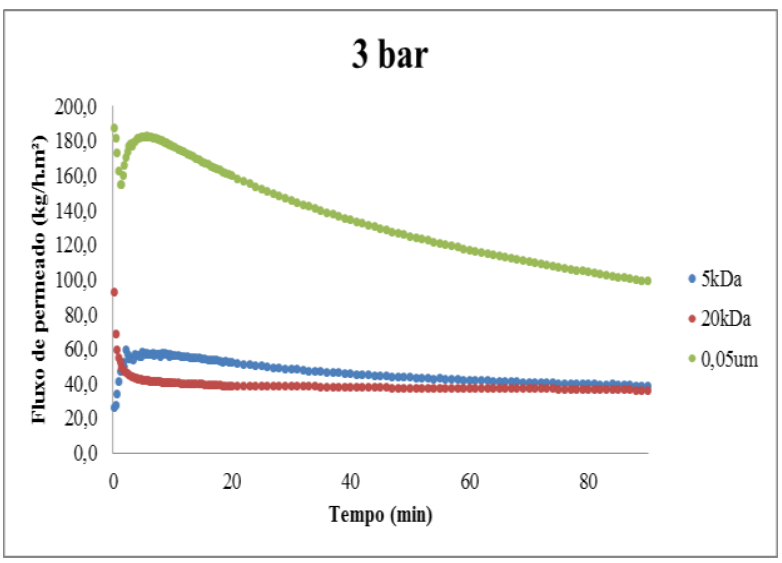

Pode-se perceber nas Figuras 5, 6 e 7 que a membrana de $0,05 \mu \mathrm{m}$ apresenta um fluxo mais elevado que as outras membranas, isso pode ser explicado pelo fato de ter diâmetro de poros maior e com isso a saída do permeado é maior.

Percebe-se também que a membrana de $0,05 \mu \mathrm{m}$ não atinge um equilíbrio no fluxo de permeado, ou seja, o fluxo diminui continuamente durante os 90 minutos de experimento. Isso pode ser explicado pelo fato de que uma diâmetro de poros maior favorece $\mathrm{o}$ entupimento dos poros da membrana, como estudado por Amin et al. (2010).

Analisando as membranas de $5 \mathrm{kDa}$ e $20 \mathrm{kDa}$ percebe-se que a membrana de $20 \mathrm{kDa}$, 
que tem um diâmetro maior de poro, apresenta um fluxo menor, oque não era esperado, porém isso pode ser explicado pelo fato da membrana de $5 \mathrm{kDa}$ ser multicanal e com isso possuir uma área maior, possibilitando uma passagem maior de permeado.

Após cada purificação foi realizado um balanço de massa do processo para verificar a quantidade de glicerol, considerando a alimentação, que passou pela membrana e o que ficou retido por ela. $\mathrm{Na}$ Tabela 2 estão os resultados desse balanço.

Tabela 2 - Quantidade de glicerol no permeado e concentrado, (\%).

\begin{tabular}{lccc}
\hline Membrana & $\begin{array}{c}\text { Pressão } \\
\text { (bar) }\end{array}$ & $\begin{array}{c}\text { Permeado/ } \\
\text { Alimentado }\end{array}$ & $\begin{array}{c}\text { Concentrado/ } \\
\text { Alimentado }\end{array}$ \\
\hline \multirow{3}{*}{$5 \mathrm{kDa}$} & 1 & 15 & 87,1 \\
& 2 & 19,4 & 74,6 \\
& 3 & 18,7 & 89,6 \\
\hline \multirow{3}{*}{$20 \mathrm{kDa}$} & 1 & 3,9 & 89,4 \\
& 2 & 5,7 & 87,1 \\
& 3 & 5,2 & 88,2 \\
\cline { 2 - 3 } $0,05 \mu \mathrm{m}$ & 1 & 14,5 & 77,6 \\
& 2 & 20,6 & 75,7 \\
& 3 & 20,1 & 75,8 \\
\hline
\end{tabular}

Os resultados apresentados na Tabela 2 demonstram que devido ao baixo fluxo permeado e ao curto tempo de filtração a maior parte do glicerol alimentado permanece no concentrado. Para uma maior recuperação deste glicerol no permeado, seria necessário um tempo maior de ultrafiltração para que todo glicerol permeasse pela membrana.

Para uma análise da eficácia da ultrafiltração, analisaremos a seguir a concentração de glicerol no permeado e concentrado.

Na Tabela 3, estão os resultados de teor de glicerol para o permeado e concentrado, após as purificações utilizando as membras de $5 \mathrm{kDa}, 20 \mathrm{kDa}$ e $0,05 \mu \mathrm{m}$, nas pressões de 1, 2 e 3 bar.
Tabela 3 - Teor de glicerol para o permeado e concentrado.

\begin{tabular}{lccc}
\hline Membrana & $\begin{array}{c}\text { Pressão } \\
\text { (bar) }\end{array}$ & Permeado & Concentrado \\
\hline \multirow{3}{*}{$5 \mathrm{kDa}$} & 1 & $80,1 \pm 0,0$ & $80,7 \pm 0,6$ \\
& 2 & $80,1 \pm 0,2$ & $72,8 \pm 0,1$ \\
& 3 & $89,6 \pm 0,3$ & $84,6 \pm 0,5$ \\
\multirow{2}{*}{$20 \mathrm{kDa}$} & 1 & $77,9 \pm 0,8$ & $73,4 \pm 0,5$ \\
& 2 & $76,9 \pm 1,8$ & $73,0 \pm 0,6$ \\
& 3 & $77,4 \pm 0,8$ & $73,4 \pm 0,5$ \\
$0,05 \mu \mathrm{m}$ & 1 & $78,4 \pm 0,8$ & $71,7 \pm 0,9$ \\
& 2 & $80,5 \pm 0,5$ & $74,8 \pm 0,4$ \\
& 3 & $81,5 \pm 1,3$ & $74,3 \pm 1,5$ \\
\hline
\end{tabular}

Percebe-se pela Tabela 3, que houve um pequeno aumento da concentração de glicerol na maioria das soluções de permeado, visto que a concentração inicial da glicerina era 78,9 \%. Na maioria das soluções de concentrado houve uma diminuição da concentração de glicerol, que pode ser observado melhor na Figura 8.

Figura 7 - Teor de glicerol para o permeado e concentrado.

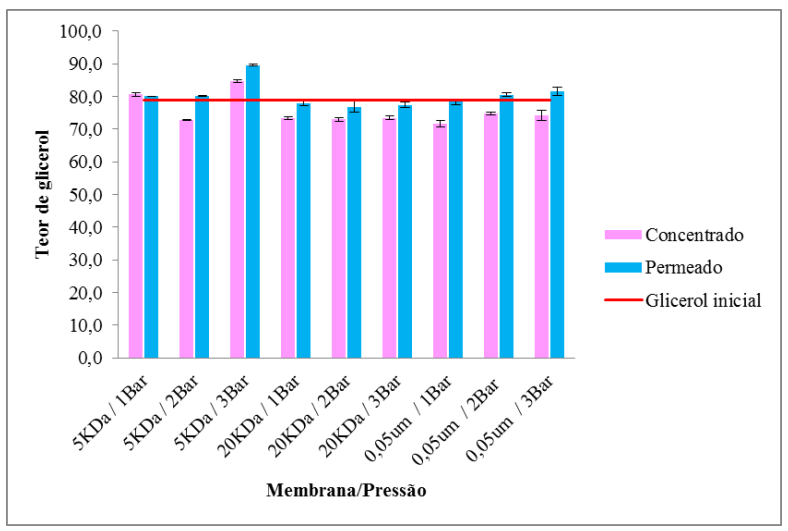

Por meio da Figura 8, podemos verificar que as membranas apresentaram uma seletividade ao glicerol, já que sua concentração foi maior no permeado quando comparada com o concentrado. O melhor resultado foi verificado para a membrana de $5 \mathrm{kDa}$ a 3 bar com $89,6 \%$ de glicerol no permeado. 


\section{CONCLUSÕES}

Nas condições em que os ensaios foram conduzidos, concluiu-se preliminarmente que, a membrana de $5 \mathrm{kDa}$ a 3 bar apresentou teor de glicerol maior no permeado. Porém até o presente momento os resultados não se mostraram conclusivos para a purificação de glicerina por processos com as membranas utilizadas.

Nas condições operacionais utilizadas no presente trabalho a purificação do glicerol por meio da ultrafiltração não atingiu valores de concentração próximos da glicerina pura. Novos estudos deverão ser realizados, utilizando outras membranas, além de alterações nas condições de operação, para se atingir maiores níveis de purificação.

\section{NOMENCLATURA}

I.A: Índice de acidez representado pela unidade: miligramas de $\mathrm{KOH} /$ gramas de amostra.

V: Volume da solução de hidróxido de sódio gasto na titulação das amostras $(\mathrm{ml})$.

$\mathrm{V}_{\mathrm{b}}$ : Volume da solução de hidróxido de sódio gasto na titulação do branco $(\mathrm{ml})$.

M: Molaridade da solução de hidróxido de sódio.

W: Massa da amostra (g)

MM $_{\text {glicerol: }}$ massa molar do glicerol, 92,09 $\mathrm{g} / \mathrm{mol}$

$\mathrm{N}$ : concentração da solução de $\mathrm{NaOH}$ (mol/L)

f: fator de correção da solução de $\mathrm{NaOH}$

$\mathrm{V}_{1}$ : volume de $\mathrm{NaOH}$ gasto na titulação da amostra (L)

$\mathrm{V}_{2}$ : volume de $\mathrm{NaOH}$ gasto na titulação do branco $(\mathrm{L})$

M: massa de amostra utilizada (g)

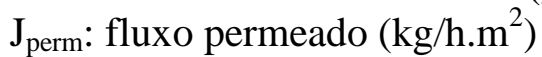

$\mathrm{m}_{\mathrm{p}}$ : massa de permeado $(\mathrm{kg})$

$\mathrm{t}$ : intervalo de tempo (h)

A: área de permeação da membrana $\left(\mathrm{m}^{2}\right)$

\section{REFERÊNCIAS}

AMIN, I. N. H. M.; MOHAMMAD, A. W.; MARKOM, M.; PENG, L. C. Effects of palm oil-based fatty acids on fouling of ultrafiltration membranes during the clarification of glicerina rich solution. Journal of Food Engineering, v. 101, p. 264-272, 2010.

ARRUDA, V.; RODRIGUES, R.; FELIPE, A. Glicerol: um subproduto com grande capacidade industrial e metabólica. Revista Analytica, n. 26, p. 56-62, 2007.

AYOUB, Muhammad, ABDULLAH, Ahmad Zuhairi. Critical review on the current scenario and significance of crude glycerol resulting from biodiesel industry towards more sustainable renewable energy industry.

Renewable and Sustainable Energy

Reviews, v. 16, p. 2671-2686, 2012.

FERREIRA, M. de O. Purificação da glicerina bruta obtida a partir da transesterificação do óleo de algodão. Dissertação de mestrado. Universidade Federal do Rio Grande do Norte, Natal, 2009.

GOMES, M. C. S.; ARROYO, P. A.; PEREIRA, N. C. Influence of acidified water addition on the biodiesel and glycerol separation through membrane technology. Journal of Membrane Science. v. 431, p. 28-36, 2013.

HÁJEK, M.; SKOPAL, F. Treatment of glycerol phase formed by biodiesel production. Bioresource Technology, v. 101, p. 3242-3245, 2010.

KNOTHE, G. Dependence of biodiesel fuel properties on the structure of fatty acid alkyl esters. Fuel Processing Technology, v. 86, p. 1059-1070, 2005. 
KNOTHE, G.; VAN GERPEN, J.; KRAHL, J.; RAMOS, L. P., Manual de biodiesel, Ed. Edgard Blucher, São Paulo, 2006.

LOPES, A. P.; CANESIN, E. A.; SUZUKI, R. M.; TONIN, L. T. D.; PALIOTO, G. F.; SEIXAS, F. L. Purificação de Glicerina Residual Obtida na Produção de Biodiesel a Partir de Óleos Residuais. Rev. Virtual Quim, v. 6, p. 1564-1582, 2014.

MOTA, C. J. A.; SILVA, C. X. A.; GONÇALVES, V. L. C. Gliceroquímica: novos produtos e processos a partir da glicerina de produção de biodiesel. Química Nova, v. 32, $\mathrm{n}^{\circ}$ 3, 2009.

MOTA, C. J. A.; SILVA, C. X. A.; GONÇALVES, V. L. C. Gliceroquímica: novos produtos e processos a partir da glicerina de produção de biodiesel. Química Nova, v. 32, n 3, 2009.

NAVIGLIO, D. Rapid determination of esterified glycerol and glycerides in triglyceride fats and oils by means of periodate method after transesterification. Food Chemistry, v. 102, p. 399-405, 2007.

OOI, T. L. et al. Glycerol residue: a rich source of glycerol and medium chain fatty acids. Journal of Oleo Science, v. 53, n. 1, p.29-33, 2004.

ROCHA, R. P. Processos de purificação/valorização do glicerol proveniente da produção de biodiesel. (Mestrado em Engenharia Química) Instituto superior de engenharia do porto, 2009.

SANTOS, A. F. Novas perspestivas da glicerina-Síntese de novos nitratos com propriedades farmacológicas e melhoradores de cetano. Dissertação de mestrado. Universidade Federal da Paraíba. João Pessoa, 2009.
TAN, H. W.; ABDUL AZIZ, A. R.; AROUA, M. K. Glycerol production and its applications as a raw material: A review. Renewable and Sustainable Energy Reviews, v. 27, p. 118-127, 2013.

VIESSER, R.V. Purificação da glicerina obtida a partir da transesterificação do óleo de girassol semi-refinado. Trabalho de conclusão de curso, Universidade Tecnológica Federal do Paraná. Curitiba, 89 pag., 2010.

VISENTAINER, J. V., JUNIOR, O. de O. S. Produção e controle de qualidade do biodiesel de óleos e gorduras. Maringá: Eduem, 2013.

\section{AGRADECIMENTOS}

O presente trabalho foi realizado com o apoio do Conselho Nacional de Desenvolvimento Científico e Tecnológico $\mathrm{CNPq}$ - Brasil, deixamos nosso sincero agradecimento. Agradecemos também à empresa BSBIOS pela doação de glicerina. 